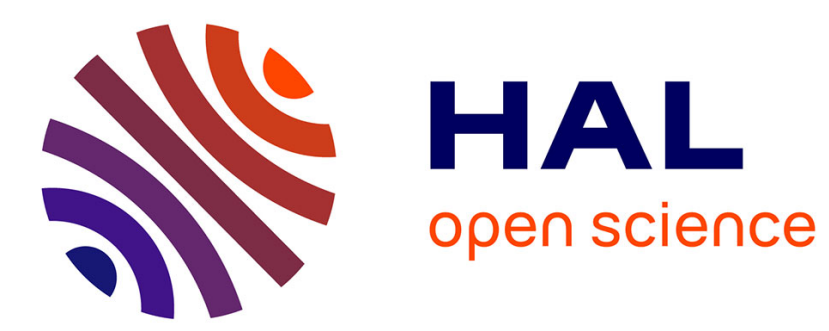

\title{
Simple traffic model for a simple problem: sizing travel time measurement devices
}

\author{
C. Buisson
}

\section{To cite this version:}

C. Buisson. Simple traffic model for a simple problem: sizing travel time measurement devices. Transportation Research Record, 2006, n1965, p210-218. hal-00505757

\section{HAL Id: hal-00505757 https://hal.science/hal-00505757}

Submitted on 26 Jul 2010

HAL is a multi-disciplinary open access archive for the deposit and dissemination of scientific research documents, whether they are published or not. The documents may come from teaching and research institutions in France or abroad, or from public or private research centers.
L'archive ouverte pluridisciplinaire HAL, est destinée au dépôt et à la diffusion de documents scientifiques de niveau recherche, publiés ou non, émanant des établissements d'enseignement et de recherche français ou étrangers, des laboratoires publics ou privés. 


\section{A Simple Traffic Model for a Simple Problem: \\ the Case of Sizing Travel Time Measurement Devices}

\section{Christine BUISSON}

Laboratoire Ingénierie Circulation Transport LICIT (ENTPE / INRETS)

Rue Maurice Audin

F 69518 Vaulx en Velin

Tel: 33472047713

Fax: 33472047712

Email: christine.buisson@entpe.fr

March 30 $0^{\text {th }}, 2006$.

Word count: 5,608 words +7 tables and figures $=7,358$. 


\begin{abstract}
As the capabilities of Automated License Plate Recognition (ALPR) devices increase, their applicability to measure individual travel times becomes appealing and the question of sizing the total number of devices becomes an important one.

ALPR cameras have been deployed along a road of about $50 \mathrm{~km}$ in the French Alps. This road is highly congested and users experiment travel times varying from 35 up to 120 minutes. Traffic managers plan to inform road users of their travel times on Variable Message Signs (VMS).

Slicing the complete journey into small sections (between two cameras) allows us to be more reactive to sudden changes in travel times. This is especially true if those changes are due to a congestion occurring at the beginning or in the middle of the journey. On the other hand, increasing the total number of cameras is not cost effective and must be avoided as much as possible.

This paper presents the formulation and results of a simplified model for studying the impact of the total number of cameras on the precision of displayed travel times. The model was kept as simple as possible. Maximum use of the few amounts of data available was made. Comparison with on-site preliminary results proves that our model gives robust results.
\end{abstract}




\section{INTRODUCTION}

Travel time information now is an operational and effective tool in traffic flow management. This is mainly due to the increasing demand for travel time information by road users and to the availability of various devices that can effectively provide such information. For a review of existing technical methods permitting the collection of individual travel times measurements, one will usefully read (1). In this reference, Turner drew up a list of six different methods, including the method used in our paper: automatic video license plate matching. In his conclusion, Turner listed advantages of this method: accuracy of travel times is very good, costs are low to moderate, and he said that this is a field proven technology. This last point is even truer almost 10 years after the writing of his paper. He also said that the use of this technique for real-time travel information is possible. This has been effectively proven for various operational deployment of video license plate recognition (2).

But, for any types of travel time collection processes, sizing the collection system is a crucial point. Various methods have been used to solve this optimization problem. One can cite, among the most recent papers, the use of simulation (3), or the use of statistical methods (4).

In this paper we will present the development and the use of an original model, aiming at answering an optimal sizing question in the case of the use of Automated License Plate Recognition devices (ALPR). The question can be summarized as: "how many cameras do I need to provide road users with valuable travel time information?".

A few amount of quantitative information was accessible on traffic variability on the road under consideration. On the other hand, thanks to the skills of the traffic management team, a good qualitative knowledge of road conditions was available.

We decided not to use microscopic simulation models, because the lack of precise quantitative data made calibration and validation of those tools out of reach.

More of that, the individual nature of traffic measurement devices (identifying license plate by video image analysis), made the use of macroscopic models useless for determining the optimal number of ALPR.

Therefore, we built an original model which can be seen as a mesoscopic one. The model was designed to use all information available for answering this question, while kept as simple as possible.

The layout of the paper is organized as follows: first we describe the underlying question and its surrounding context; second we present various kinds of travel times and the impact those definitions may have in our context; third we explain the model and the results obtained. Model results and in-site experiment, confirming the models results, will be presented into the fourth part. 


\section{CONTEXT AND ORIGIN OF THE MODELING APPROACH}

\section{General Objective of the Project: Providing Users with Their Expected Travel Times in an Alps Valley}

\section{The specific context of a narrow alpine valley}

The Oisans Valley situated South of Grenoble is the location of two famous ski resorts: Les Deux Alpes and l'Alpe d'Huez. The demand of traffic originated by those resorts is of the order of 17,000 vehicles during a congested Saturday of winter ${ }^{1}$. The road capacity is typically of 1600-1800 vehicles per hour for this one-lane-per-direction road. As usual, congestion is caused by the particular distribution of the demand, which is concentrated on the morning. Therefore travel times are as high as 2 hours or more for 45 kilometers.

The enhancement of the road is impossible without considerable road works. Travel times information appears to be a good solution. Actually, previous experiment in urban and interurban cases reported that providing information reduced the stress of drivers even if it is inefficient in diminishing travel times.

The information displayed will consist of mean travel times, displayed on three variable message signs (VMS). The travel times will be calculated, from each VMS until Bourg d'Oisans, which is the last village of the valley before the ski resorts.

\section{Specific objective of the use of Automated License Plate Recognition in this context}

The road presents various characteristics: village crossings, ramps, curves and roundabouts and so on, each one lying at the root of a possible congestion. Figure 1 presents the simplified map of the road.

Six electro-magnetic loops are installed on this stretch of $45 \mathrm{~km}$ long. This could have been estimated as sufficient for travel time estimation. The traffic managers decided to use automated vehicle identification for various reasons:

- It is a mean to test this device in France where it is rarely used.

- The cameras of automated plate recognition are costly at the moment but the cost of it should rapidly decrease as the number of implanted devices increases.

- Another reason to use ALPR instead of inductive loops is linked to the fact that loops only allow travel times to be indirectly inferred from speed estimates at specific point while ALPR would allow a direct measurement of travel times.

\section{Insert Fig 1 here}

\footnotetext{
${ }^{1}$ Saturday is the most congested day in the week, because renting of apartments comes into weeks, from one Saturday until the next one. Tourist interest is mainly for snow season, therefore a total amount of 20 very congested Saturday per year is reasonable.
} 


\section{Determination of potential positions of the cameras and limitation of the total number}

The motivations for choosing the position of potential cameras were the following:

- Technical reasons related to transmission and building costs,

- The objective of being as near as possible from the VMS signs where information will be displayed (to ensure the maximum liability of the travel times displayed)

- And the goal of being as near as possible of the exit of existing bottlenecks.

Therefore the question we addressed was not to choose the position of the cameras, but on the contrary, to choose, among all the seven possible locations, the optimal subset. The correlative question was to determine in advance what precision could be hoped on travel times for each subset.

The more ALPR devices placed on the road, the smaller the subsections, the more reactive the systems to traffic conditions, the more correct the displayed travel times.

The simple following example will try to explain it. Suppose that the total travel time for the two subsections composing the road is of 10 minutes in normal conditions. If, at a certain time, congestion occurs in the first subsection, having a camera at the end of it allows to be averted of the occurrence of congestion 10 minutes in advance; compared with the situation with a camera at the end of the second subsection only.

The perfect solution would then have been to place a camera at the exit of each potential bottleneck. On the other hand, each ALPR is expensive and the total number of cameras has to be limited. The position of each camera has to be determined carefully, depending on the traffic condition variations between the possible positions of them.

\section{Description of the global project of travel time information}

The modeling work reported here is a part of the project carried out under the supervision of Direction Départementale de l'Equipement de l'Isère, which is the French state authority in charge of this stretch of road. This project began in late 2002 and is now in operational stage, with five cameras and effective travel time diffusion. Different stages of the project took place, realized by different yet coordinated teams.

- Choosing the vendor and measure performances of the ALPR devices (depending on the site, 70 to $80 \%$ of good identification were obtained);

- Choosing the total number and implantation of cameras (the work presented in this paper was developed specifically to help traffic managers to realize this particular task);

- Defining the information chain that leads from the identification of one single vehicle by one camera through the filtering and aggregation of travel times to the posting of travel time information. 
Accompanying the deployment of the project, a complete procedure of evaluation is devoted to determine the perception users have of the project. First results show that the users acknowledge the help given by the information system, mainly in reducing their stress.

The chain of information from the individual identification to the posting of the travel times on the VMS is presented on the Figure 2. The model was designed to reproduce each link of this chain.

\section{Insert Fig 2 here}

\section{VEHICLE PROGRESSION MODELLING AND DEVICE EVALUATION}

\section{Requirements for a Traffic Model in the Case of Sizing Individual Measurement Devices}

The functionalities that the model must have in the special case of defining the number of Automated License Plate Recognition systems are the following:

- Modeling vehicles individually, in order to be able to:

- reproduce the dispersal of individual behaviors (inclination to overtake the leading vehicle for example)

- determine precisely the passing time of them in front of each camera,

- simulate various inaccuracies of the ALPR system (ability to identify or not a vehicle and possibly misreading one of the characters);

- Reproducing variation of total flow and of origin destination patterns in the network. Indeed we know that the measurement frequencies will have an influence on the ability of the system to capture travel time variations and that the OD matrix will modify the number of vehicles which can be seen by the successive cameras in the network;

- Varying travel times depending on the hour of the day and of the type of day.

\section{Existing Models}

Various types of models exist; the reader is referred to (5) for example for a more detailed description of them and insight about the numerous literature available on the subject. One can distinguish two different categories:

- Some are microscopic and consider each vehicle individually. The progress of the vehicle, its speed and position at each time, are explained by its desired speed, its stopping distance, the distance between it and the vehicle behind, the speed of the leading vehicle, the presence of road signs ...

- Some are macroscopic and suppose that traffic flow can be considered as a continuous flow, and therefore be simulated on the basis of hyperbolic conservation equation(s) that one can solve numerically. 
Models of this last group have been eliminated because they impede the tag of vehicles along their progression in the network. This would have made impossible the simulation of the impact of the number and efficiency of the cameras.

Microscopic models also have been rejected. Those models are designed for individual identification and their use could have been suitable for this project. But the network is very compound, and numerous bottleneck heads are far away from loops. Another reason is that available loops traffic data are aggregated over 6 minute periods. Therefore the calibration and validation of any of the numerous microscopic models reproducing the progress of vehicles along the road would have been impossible without a large amount of time.

A closer examination of the problem showed us that a reproduction of the progress of individual vehicles along the road was not necessary for our purpose. The travel times between the positions of two successive cameras can be determined not endogenously, as in classical microscopic models, but exogenously.

Actually, no matter what happens between two successive cameras if travel times are sufficiently well reproduced. Moreover, the values of travel times in sub sections of the network can be determined from qualitative knowledge of the behaviour of the network.

Therefore, we chose to build up a completely new model, able to capture the knowledge of traffic managers as input, and to present all the features (and only those features) that are necessary for evaluating the performance of the system. This model can be seen as a mesoscopic model.

\section{Modeling the complete information chain}

\section{Entry data}

Suppose that we have to reproduce a stretch of road divided into sub sections. Two sub sections are connected together by one node. The total number of sub sections is noted $S$, and the set of the sub section can be denoted $\left\{s^{1}, s^{2}, \ldots, s^{s}\right\}$. Suppose that the duration of the simulation is divided into periods of length $\Delta t$.

The entry of the model can be expressed as the following. Suppose that, for each sub section $s$ at each period $t$ of the simulation, we know:

- $Q_{s}(t)$, the total input flow of the sub section;

- $\theta_{s}(t)$, the mean travel time of the section for all vehicles $N_{s}(t)=Q_{s}(t) \times \Delta t$ entering this sub section during this period of 1 minute.

Let recall that, where travel time is usually a result of the propagation of vehicle inside the microscopic model, in our case it is determined exogenously of the simulation model, thanks to data collected from loops, interviews with traffic managers... 


\section{Mean journey travel time}

The complexity of the problem lies in the fact that the mean journey travel time $\Theta(t)$ is not simply the sum of sub sections mean travel times. It is indeed the following non linear and recursive function (for example in the case where the road is composed of three sub sections):

$$
\Theta(t)=\theta_{s^{1}}(t)+\theta_{s^{2}}\left(t+\theta_{s^{1}}(t)\right)+\theta_{s^{3}}\left(t+\theta_{s^{2}}\left(t+\theta_{s^{1}}(t)\right)\right)
$$

This equation means that the travel time of the last section traveled depends on the travel times of the two previous sections.

\section{Sub section individual travel time determination}

Each vehicle $i$ of the $N_{s}(t)=Q_{s}(t) \times \Delta t$ vehicles entering the sub section during period $t$, will experiment an individual travel time $\tau_{s}^{i}(t)$, whose difference with the mean travel time of the sub section $\theta_{s}(t)$ can be explained by individual dispersion, aggressiveness, overtaking propensity ... Individual and mean travel times are related together, at each period and for each sub section, by the relation:

$$
\theta_{s}(t)=\operatorname{mean}\left(\tau_{s}^{i}(t)\right)=\frac{1}{N_{s}(t)} \sum_{1}^{N_{s}(t)} \tau_{s}^{i}(t)
$$

Please note that equation (2) is valid because the values $\tau_{s}^{i}(t)$ are deduced from input data in our model. In the general case, they are known only at time $t^{\prime}=t+\tau_{s}^{i}(t)$, where $t$ is the entry time and $t^{\prime}$ is the exit time of the sub section $s$.

The distribution of the desired individual travel times can be expressed as a Gaussian distribution, with a standard deviation $w$, chosen here to be proportional to the mean travel time. If overtaking on the sub section is possible, we consider that the width of the distribution will be multiplied by a factor $O$. After driving through the link, individual travel times are not the $\tau_{s}^{i}(t)$, but the experimented ones $\tau^{*}{ }_{s}^{i}(t)$. This modification is due to the respect of minimal headway $h_{\min }$, that will naturally increases the values of individual travel times $\tau^{* i}(t) \geq \tau_{s}^{i}(t)$.

The equation (2) above can now been expressed as:

$$
\theta_{s}(t) \approx \operatorname{mean}\left(\tau^{*}{ }_{s}^{i}(t)\right)
$$

where $\tau^{*}{ }_{s}^{i}(t)$ is the value of the travel time of vehicle $i$, entering sub section $s$ at minute $t$, modified by respect of minimal headway $h_{\min }$.

$\tau^{* i}{ }_{s}(t)$ reproduces in our model the physical phenomena of the first step of Figure 2. 
Journey individual travel time

It is important here to emphasize the fact that the journey individual travel time is not simply the sum of sub sections individual travel times. On the contrary, the same formula as described in equation (1) holds on the individual level:

$$
T^{i}(t)=\tau^{* i} s^{1}(t)+\tau^{* i} s^{2}\left(t+\tau^{* i} s^{1}(t)\right)+\tau^{* i} s^{3}\left(t+\tau^{* i} s^{2}\left(t+\tau^{* i} s^{1}(t)\right)\right)
$$

which significantly differ from $\tau^{* i} s^{3}(t)+\tau^{* i} s^{2}(t)+\tau^{* i} s^{1}(t)$, especially when sudden variations appear in mean travel times of the downstream sub sections. (Once again the formula was given for three sub sections only, but can easily been generalized).

Additional information needed for describing the journey of the simulated vehicles

We can estimate, from loop detectors, the secondary input flows and the diverting proportion of vehicles in each node, nodes being situated between sub sections. We can also determine, for each sub section, the possibility of overtaking or not. This possibility depends on the traffic regulations. We use all the available information to determine those values, therefore reproducing vehicle trajectories, as can be seen on the Figure 3.

\section{Insert Fig 3 here}

\section{Probability of a vehicle i to be correctly identified by ALPR devices on the sub section $\mathrm{s}$}

We reproduce the identification of vehicles as they pass in front of the cameras (step 2 in Figure 2) with the following characteristics. Some vehicles are not seen by any of the cameras, because of the dirtiness of the license plate or position of it on the vehicle. Each camera has its own probability to detect or not a vehicle $\partial$ and, if the license plate is correctly photographed. The probability that the video imaging algorithm miss-read one of the eight characters of the license plate is denoted $r$.

All those probabilities are introduced in our model, which give us the passing time in front of both cameras of the sub section $s$ at each minute $t$, for all simulated vehicles identified.

Delay due to the transmission system

Cameras give individual values (instant of passing in front of the cameras). Transmission delay (noted TD) of those values is at the moment as long as 6 minutes. The update frequency of the ALPR devices is of 3 minutes and noted UF. This has a significant impact on the reactivity of the system and has to be reproduced (step 3 of Figure 2). 


\section{Reproduction of the central node}

We turn now to the fourth step of the information chain. Calculations are made on a central computer receiving all information transmitted by the ALPR devices.

Matching passing time between two successive cameras is easy and has no impact on the quality of calculated travel times.

The filtering and aggregating travel times procedure is described in (O). This procedure has to be completely reproduced in the simulation, because the modifications introduced in the evaluation of mean travel times for each sub section can affect the final result.

The purpose of the filtering is to remove abnormal travel time values: some of the vehicle stops for a pause or take a shortcut ${ }^{2}$; miss reading of license plate can leads some pairs to be made from two distinct vehicles.

The purpose of aggregating is to diminish the impact of individual variations.

Filtering and aggregating of individual travel times are made on a fixed sample size basis. It means that each time an update of information is made, the travel times of last $n$ vehicles exiting the link are filtered and the mean travel time is computed.

Note that this method is more reactive than fixed time interval which considers all the vehicles exiting the link during the previous period of a given length. Actually, when travel times vary quickly, total flow is usually high and the period of time necessary for collecting a sufficient number of vehicles is shorter than in period of lower values of travel time, flow and travel time variations.

The filter parameters for the simulation used in this paper are identical to the one used in the effective deployment of the system. The number $n$ of individual travel times used to compute a mean value is 30 .

The filter takes as entry, for each sub section and for each period $t$ ' ' of length UF, the $n$ individual travel times that have been transmitted recently. If $T D$ is the transmission delay, then the exit period of those vehicles is $t^{\prime}$ or before, with

$$
t^{\prime \prime}=t^{\prime}+T D
$$

In turn, for a vehicle exiting at period $t^{\prime}$, the entry period is related with the individual travel time by the relation:

which gives:

$$
t^{\prime}=t+\tau^{* i} s^{1}(t)
$$

$$
t^{\prime \prime}=t+\tau^{* i} s^{1}(t)+T D
$$

\footnotetext{
${ }^{2}$ A shortcut, known by approximately $15 \%$ of the total traffic (local users) is used when congestion occurs. Its travel time is of 7 minutes (constant), where on the corresponding subsection travel time varies form 3 to 20 minutes.
} 
Therefore, the mean value computed by our filter for the period $t^{\prime \prime}$, denoted $\hat{\theta}_{s}\left(t^{\prime \prime}\right)$, can be significantly different from $\theta_{s}\left(t^{\prime \prime}\right)$, the mean sub-section travel times for vehicles entering the sub section at time $t^{\prime \prime}$. Reasons explaining this difference are the following:

- The $\hat{\theta}_{s}\left(t^{\prime \prime}\right)$ mean travel times are not those used at the entry of the model $\left(\theta_{s}(t)\right)$, but an estimation of it,

- The period $t^{\prime \prime}$ corresponds to an exit time plus transmission delay and not to the entry time $t$.

- This has to be completed by the remark that the estimate of mean travel time $\hat{\theta}_{s}(t)$ is constant during a period of length UF equal to the update frequency of information.

For a given route, adding sub-section estimates of travel times $\hat{\theta}_{s}\left(t^{\prime \prime}\right)$ leads us to an estimation of the travel time of this road, noted $\hat{\Theta}\left(t^{\prime \prime}\right)$, and computed on the transmitted information of last exited vehicles of each sub sections. Two remarks concerning this route travel time:

- It is computed by summing up sub sections travel times $\hat{\theta}_{s}\left(t^{\prime \prime}\right)$ of different vehicles; i.e., the probability that a given vehicle exits from all the sub sections composing the road among the $n$ vehicles used to compute each sub section mean travel time is very low.

- This travel time $\hat{\Theta}\left(t^{\prime \prime}\right)$ is posted on the VMS signs at the beginning of the period $t^{\prime \prime}$. Therefore when the user sees the information on the VMS, this information is an observation of what happened a significant amount of time before.

Figure 4 illustrates a simple case. Vehicles are identified while passing in front of the three cameras: $\mathrm{C} 1, \mathrm{C} 2$ and $\mathrm{C} 3$. The sub section 1 is limited by cameras $\mathrm{C} 1$ and $\mathrm{C} 2$, the sub section 2 , by $\mathrm{C} 2$ and $\mathrm{C} 3$. The estimate of the mean route travel time $\hat{\Theta}\left(t^{\prime}\right)$ is displayed on the VMS at time $t^{\prime}$. This value is the sum of the mean travel time of the $n$ last exited vehicles of the two sub sections (here, for illustrative purpose, only two vehicles are used to compute mean value in each sub section). The first vehicle entering the road at time $t^{\prime}$ (vehicle E) will experiment the travel time $T^{E}\left(t^{\prime}\right)$. Please note that here all vehicles A to D entered all sub sections during time period $t$, but that this not usually the case. Here we have taken $\mathrm{TD}=0$ (no transmission delay).

Evaluating the quality of the information delivered on VMS toward road users

At the moment, the information delivered to road users is the estimate of road travel time $\hat{\Theta}\left(t^{\prime \prime}\right)$, for each route beginning with a VMS sign and ending in Bourg d'Oisans, the most distant village in the valley, before winter sport resorts. 
We have two possible ways of evaluating the information chain described above and reproduced by our simulation model:

- The first way is to evaluate how much this chain modifies the mean journey travel time, this means comparing $\hat{\Theta}(t)$ (estimate of mean route travel time) and $\Theta(t)$. This last value is the real value in the case of on-site experiment. Note that $\Theta(t)$ can not be known precisely. Thanks to the use of simulation, we know in our case this value exactly, because this is an entry data of our model. Therefore, provided that our simulation reproduces correctly the information chain, we are able to evaluate the effect of this chain on the estimate of journey travel time.

- The second way is a more complete one, because it allows mimicking the fact that road users take the travel time value displayed on the VMS as a prediction of their own travel time. Once arrived in Bourg d'Oisans, they compare the travel time they have incurred with the value they read on the VMS. Therefore, we have to compare on the one hand, the set of individual journey travel times for vehicles entering the network at time $t:\left\{T_{i}(t)\right\}$ and on the other hand the estimate of mean route travel times $\hat{\Theta}(t)$, that was displayed on the VMS when those vehicles entered the network at time $t^{\prime \prime}$, related with $t$ by mean of equation (7): $t^{\prime \prime}=t+\tau^{* i} s^{1}(t)+T D$. Please remark here that comparing the set $\left\{T_{i}(t)\right\}$ with the unique and mean value $\hat{\Theta}(t)$ must be made through distribution analysis.

We have chosen this second way because it allows us for evaluating the ability of the system to provide road users with information they really want to benefit from. This is illustrated by Figure 4 , especially by comparing the travel time $T_{E}\left(t^{\prime}\right)$ incurred by the vehicle $E$, and the travel time displayed at the entry period of this vehicle $\hat{\Theta}\left(t^{\prime}\right)$.

\section{Insert Fig 4 here}

\section{Conclusion about entry values and chosen parameters}

Entry values are composed of the description of the flowing conditions: total flow and travel times for each minute for each sub section. Those values are taken from the knowledge of the network, and from the loop detectors data. Description of the measurement device is made by defining the position and number of cameras at entry and exit of sub sections. 
Parameters reproduce constraints and characteristics of the system:

- Progression of vehicles along the sub sections:

- $\quad w$ : width of individual dispersion of travel times around the mean sub sections travel times $(2 \%)$

- 0 : sub section multiplying factor when overtaking is permitted (3 times, which means that the width of dispersion of individual travel times is of $6 \%$ );

- $h_{\min }$ : minimal headway between two successive vehicles $(0.8 \mathrm{sec})$;

- Identification of vehicles by ALPR devices (values chosen in agreement with on-site measurements of cameras performances):

- Position and number of ALPR devices;

- $d$ : probability of each vehicle to be seen by every camera $(5 \%)$;

- $\partial$ : probability of each of the camera to see one vehicle (15\%);

- $r$ : probability of each camera to modify one of the eight characters of each license plate $(5 \%$;)

- Interrogation of ALPR by the central computer:

- $T D$ : transmission delay (6 minutes);

- UF : Frequency for interrogating ALPR devices (3 minutes);

- Filtering of individual measured values to compute mean sub section travel times between two cameras:

- $n$ : number of sub sections individual measured travel times used to compute mean sub sections travel times $(n=30)$;

- filtering parameters (they are described in (6)).

- Posting of estimated travel times on the VMS (chosen to be realistic):

- Position of the VMS signs;

- Displaying strategies.

\section{RESULTS}

\section{Verification of the Role of Stochasticity}

Vehicles generation and progression along the road are determined according to stochastic distributions. Therefore, the analysis of the results must contain a phase devoted to evaluating the impact of the stochasticity. It was proven that the impact of the stochasticity was on the order of the travel time's standard deviation.

\section{Results of the Model}

\section{Choosing the Measure of Effectiveness}

How can we evaluate travel time quality of information for a road user entering the network? Users do not use relative values, but absolute ones and the comparison they make is between 
the value given at the entrance and the travel time they finally realized. We choose, therefore, to use discrepancies expressed in minutes for evaluating the information displayed in different configurations of the system.

Moreover, not only the absolute value but the sign of the difference can be important: if displayed travel time is significantly smaller than the individual one, the user will see the difference, and this can diminish the confidence he/she has in travel time information. On the contrary, if the displayed travel time is greater than the realized individual one, then the user will enjoy it, and this will less affect his/her confidence. This last effect is small and we chose not to evaluate it.

\section{Prevision of the model concerning the impact of the number of cameras}

We chose the realistic parameters defined above for describing the ALPR, transmission devices, filtering and aggregation procedure and displaying process. Those values were chosen in close collaboration with traffic managers and consultants ( 6 ).

Multiplying the total number of cameras is useful provided that they are located in positions where they allow capturing the congestion phenomena the system could otherwise ignores. The traffic scenarios and the total days those scenarios occur are therefore important. If we take a congested day, for example, the impact of the total number of the cameras is illustrated on Figure 5.

\section{Insert Fig 5 here}

This qualitative information is usefully completed with the Table 1 which presents the results obtained for various traffic conditions and various numbers of cameras. One sees again that a number of cameras lower or equal to three, is not adequate as it leads to a high proportion of travel time discrepancies exceeding 15 minutes during congested or heavily congested day. On the contrary, results are correct for 5 and 4 cameras. For 6 or 7 cameras, same results as for 5 cameras are obtained, except in the case of heavily congested Saturdays (occurring less than 4 times a year). In those cases, an extra camera reduces the number of people having a discrepancy bigger than 10 minutes of an amount of $1 \%$ per extra camera.

\section{Insert Tab 1 here}


Comparison of Simulated Accuracy of the System and Observed Accuracy on a subpart of the network

The system is now fully operating but we do not dispose at the moment of the complete data. This allowed us to make a partial evaluation of the predictions presented above.

We will here use information from the first stage of deployment of ALPR: in spring 2004, the measurement system was deployed over the first part of the network (from Pont de Claix until Vizille) with three cameras (Drac, Champagnier and Cornage, see Figure 1), covering the first 9 kilometers of the network. This part of the network is the most intensively used and therefore the most congested of the road.

We used the model presented above to predict efficiency with 2, 3 or 4 cameras for this part of the network. Results conducted the traffic managers to install three cameras. Indeed, accuracies predicted for two cameras only did not seem being acceptable.

The predicted percentage of vehicles with a more than 10 minute gap between experimented and displayed travel times are presented on the Table 2 for one scenario, corresponding to a congested Saturday. We present also the results obtained from on site data using the three cameras or only two of them on the Saturday $10^{\text {th }}$ of April 2004 which present a congestion pattern similar to this two traffic scenarios.

Insert Tab 2 here

One can see for this particular day a good agreement between observed and predicted effect of the third camera in relative values. The fact that experimental results are better than simulated ones is due to differences between two scenarios, travel times variations in the simulated case being more important than in the experimental one.

Those results prove that our model can be used with good confidence in the case of sizing measurement devices. 


\section{CONCLUSION}

In this paper we describe the building blocks of a model devoted to the estimation of the precision of travel times delivered to road users and estimated by mean of Automated License Plate Recognition (ALPR) devices. The sizing of the measurement system and the determination of the total number of ALPR imply that the model is able to reproduce as well the individual travel times dispersion and evolution; as all successive transformations from those individual values until the displaying of mean travel times on variable message signs.

The model is a mesoscopic model; which takes as input all available information from data concerning the travel conditions of vehicles.

The paper presents various simulation results and proves from on-site measurements that the results are robust.

Further improvements can be done on the operational device to shorten the transmission delays. Also, developing a new filtering and aggregating algorithm will offer not only to display some measured estimations of road travel times, but also to use prediction of travel times. The large amount of individual travel times that is collected during the winter 2006 will permit to calibrate the prediction algorithm.

\section{ACKNOWLEDGEMENTS}

The author whishes to thank warmly Fabrice Gleize from Direction départementale de l'Isère, who gave her the opportunity to work in this stimulating project. Acknowledgments are also directed towards all the members of the project team: Nathalie Rabat (DDE de l'Isère), Catherine Barthe and Patrick Olivero (Zone Expériementale de traffic de Toulouse), Gildas Lemaître (CETE Méditerannée) and Stéphane Viannez (CETE de Lyon).

The author wants to acknowledge the work of the anonymous referees who carefully analyzed the first stage of this paper. Also warm thanks are due to Jorge Laval for its contribution revising the manuscript and useful discussions. 


\section{REFERENCES}

1. Turner, S.M., Advanced Techniques for Travel Time Data Collection. In Transportation Research Records, No. 1551, 1996. p. 51-58.

2. Park, B., J. Won, and I. Yun. Application of Microscopic Simulation Model Calibration and Validation Procedure: Case Study of Coordinated Actuated Signal System. in Transportation Research Board. 2006. Washington.

3. Chen, M. and S.I.J. Chien, Determining the number of probe vehicles for freeway travel time estimation using microscopic simulation. In Transportation Research Records, No., 2000. p. 61-68.

4. Matthew W. Green, Michael D. Fontaine, and B.L. Smith, Investigation of dynamic probe sample requirements for traffic condtion monitoring. In Transportation Research Records, No. 1870, 2004. p. 55-61.

5. Gartner, N., C.J. Messer, and A.K. Rathi, eds. Traffic Flow Theory: A state of the art repport. Vol. 2000. 2000, Transportation Research Board: Washington.

6. Buisson, Ch., Affichage des temps de parcours dans la vallée de l'Oisans : Simulation de l'implantation complémentaire de caméras, LICIT, Editor. 2005, Licit: Bron. p. 75. 
List of Figures:

Figure 1: Simplified map of the road from Pont de Claix to Bourg d'Oisans. The roundabout of Champagnier corresponds to an important entry (30\% of vehicles); the crossing of Cornage to an important exit of flow (40\%).

Figure 2: Chain of information from the passing of a vehicle in from of the cameras until the posting of mean travel time information on the VMS.

Figure 3: Progression of vehicles presented on a $(x, t)$ plane. Three sub sections are presented. In the first two ones overtaking is impossible, whereas it is possible in the third one. Open circles correspond to vehicles exiting the road; black dots correspond to vehicles entering the road.

Figure 4: Representation, in the $(\mathrm{x}, \mathrm{t})$ plane, of the trajectory of some vehicles. .................... 22

Figure 5: Results of simulation for a congested Saturday for the road travel time from Pont de Claix until Bourg d'Oisans (top); the bottom figure illustrates the distribution of discrepancies between individual realized travel times and displayed travel times for the same case. 


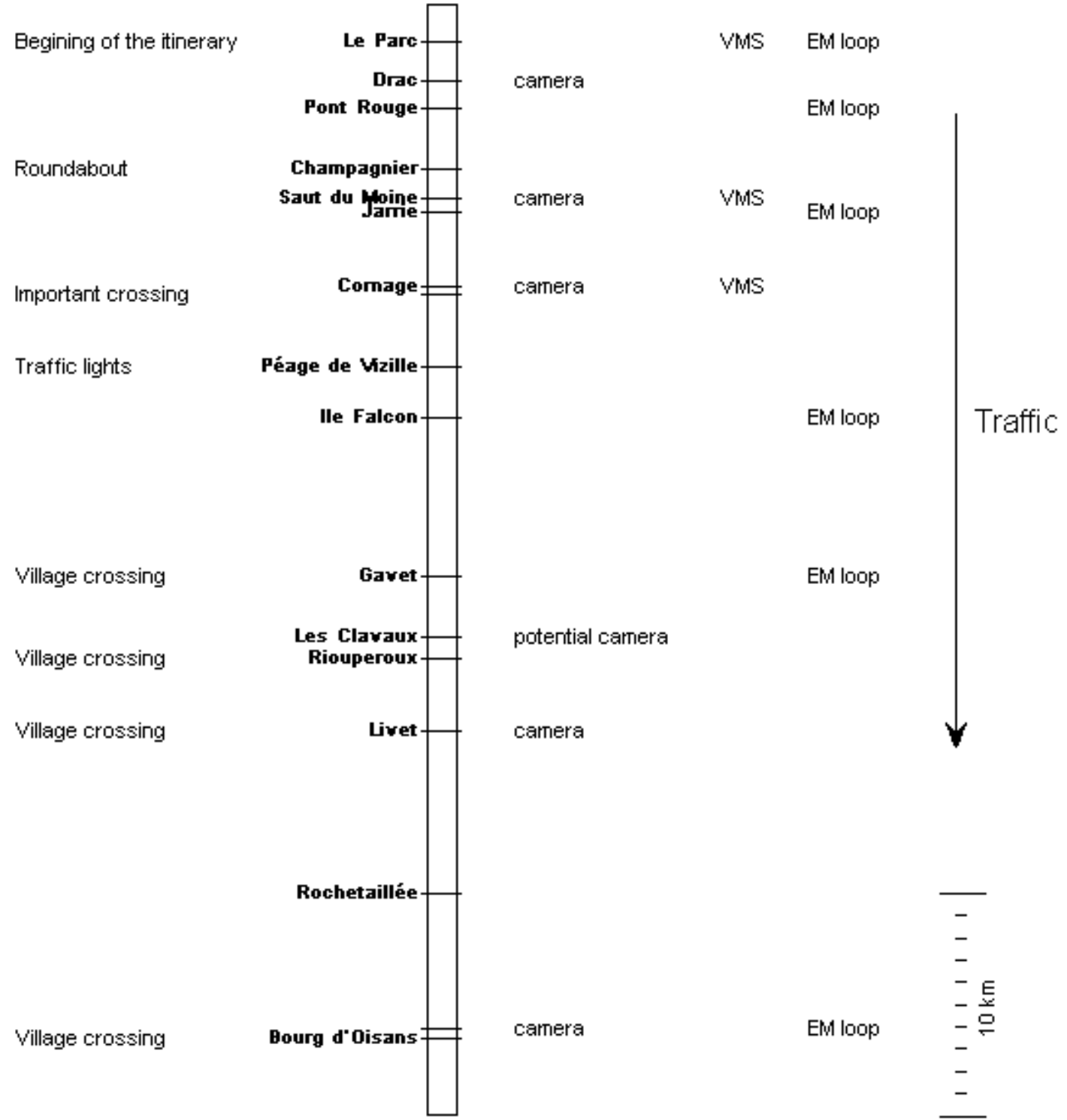

Figure 1: Simplified map of the road from Pont de Claix to Bourg d'Oisans. The roundabout of Champagnier corresponds to an important entry ( $30 \%$ of vehicles); the crossing of Cornage to an important exit of flow $(40 \%)$. 


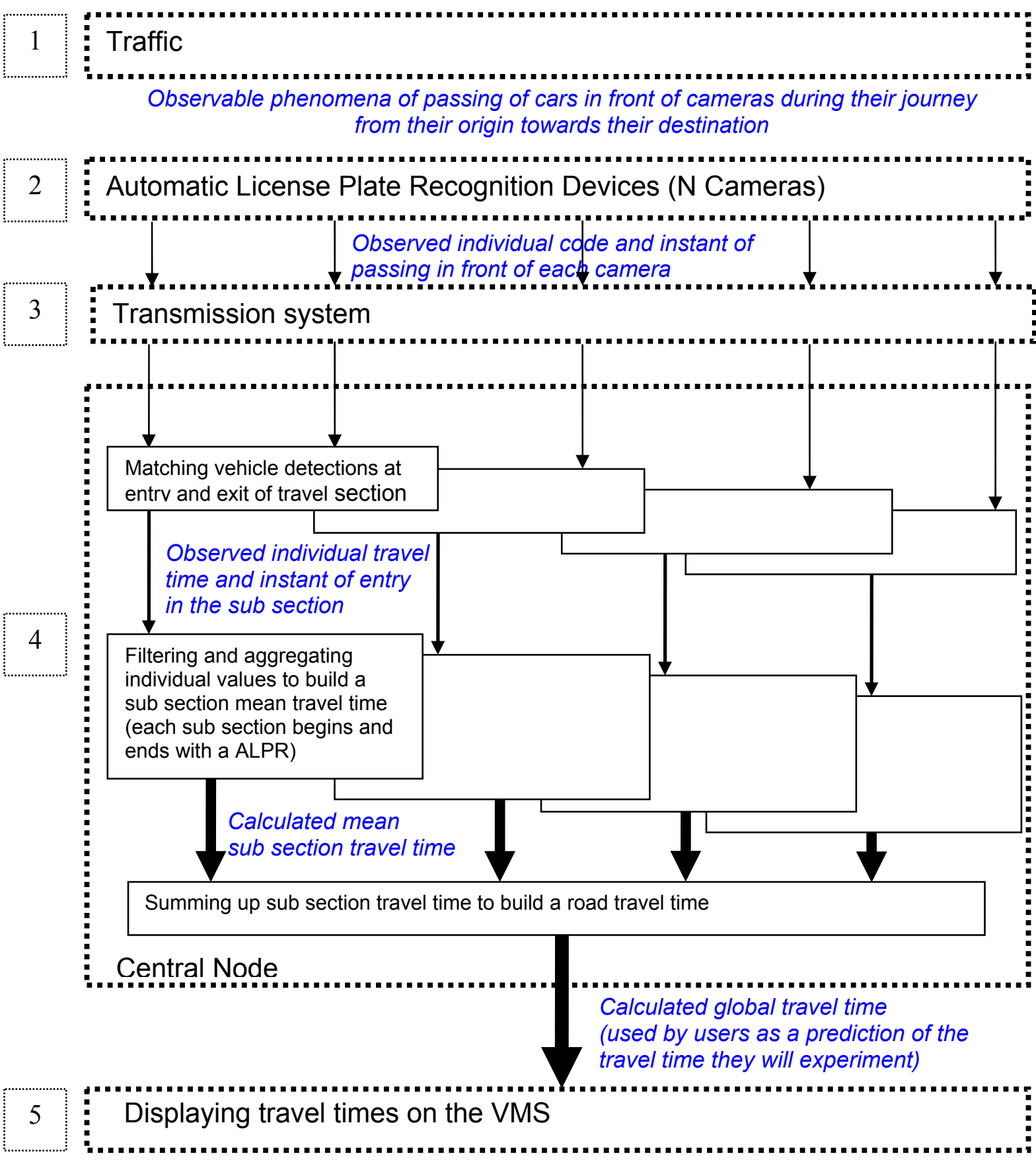

Figure 2: Chain of information from the passing of a vehicle in from of the cameras until the posting of mean travel time information on the VMS. This chain contains five successive links. Each link leads to specific information which is in turn used by the successive link. The bias introduced into information by each link must be reproduced by the traffic model. 


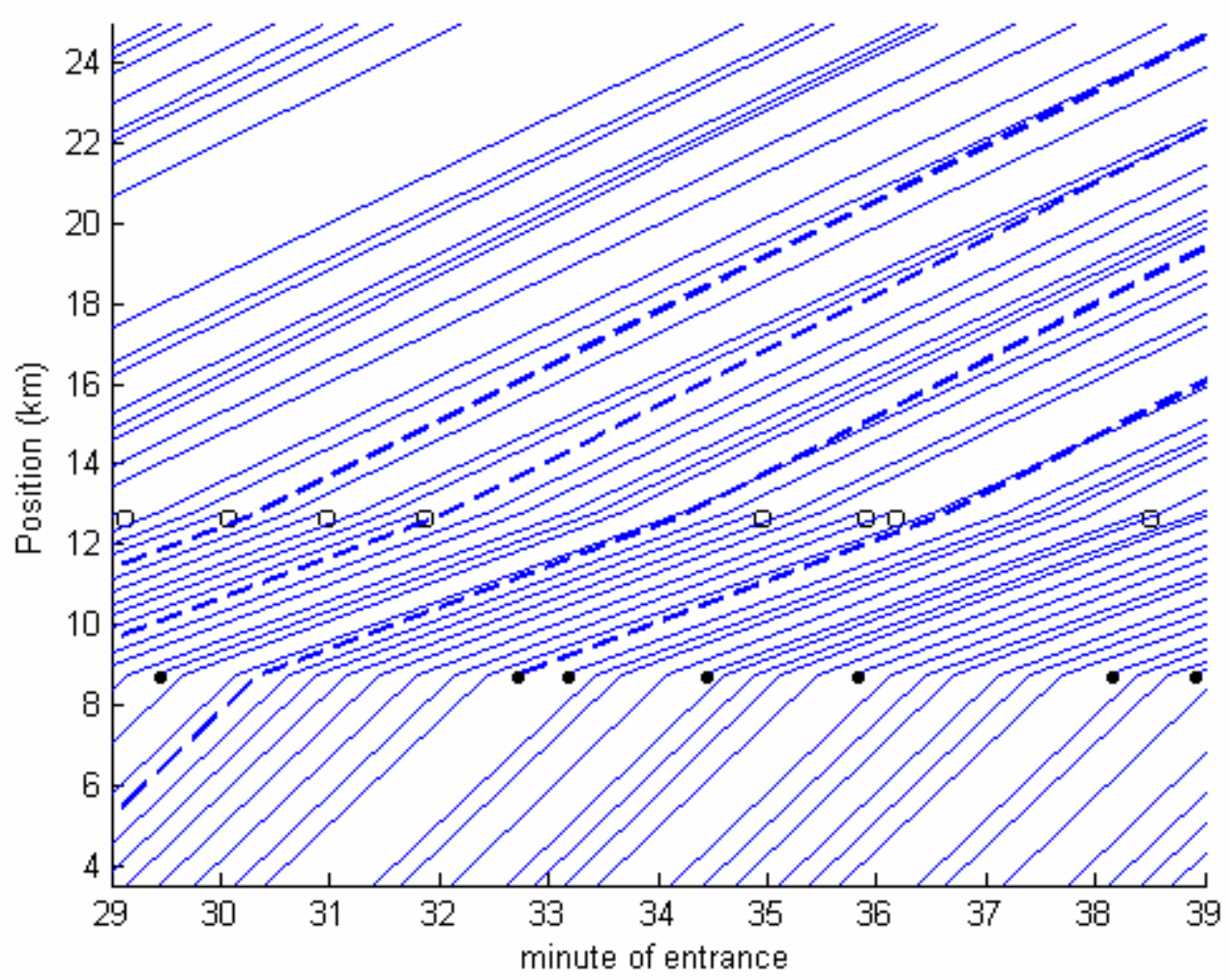

Figure 3: Progression of vehicles presented on a $(x, t)$ plane. Three sub sections are presented. In the first two ones overtaking is impossible, whereas it is possible in the third one. Open circles correspond to vehicles exiting the road; black dots correspond to vehicles entering the road. 


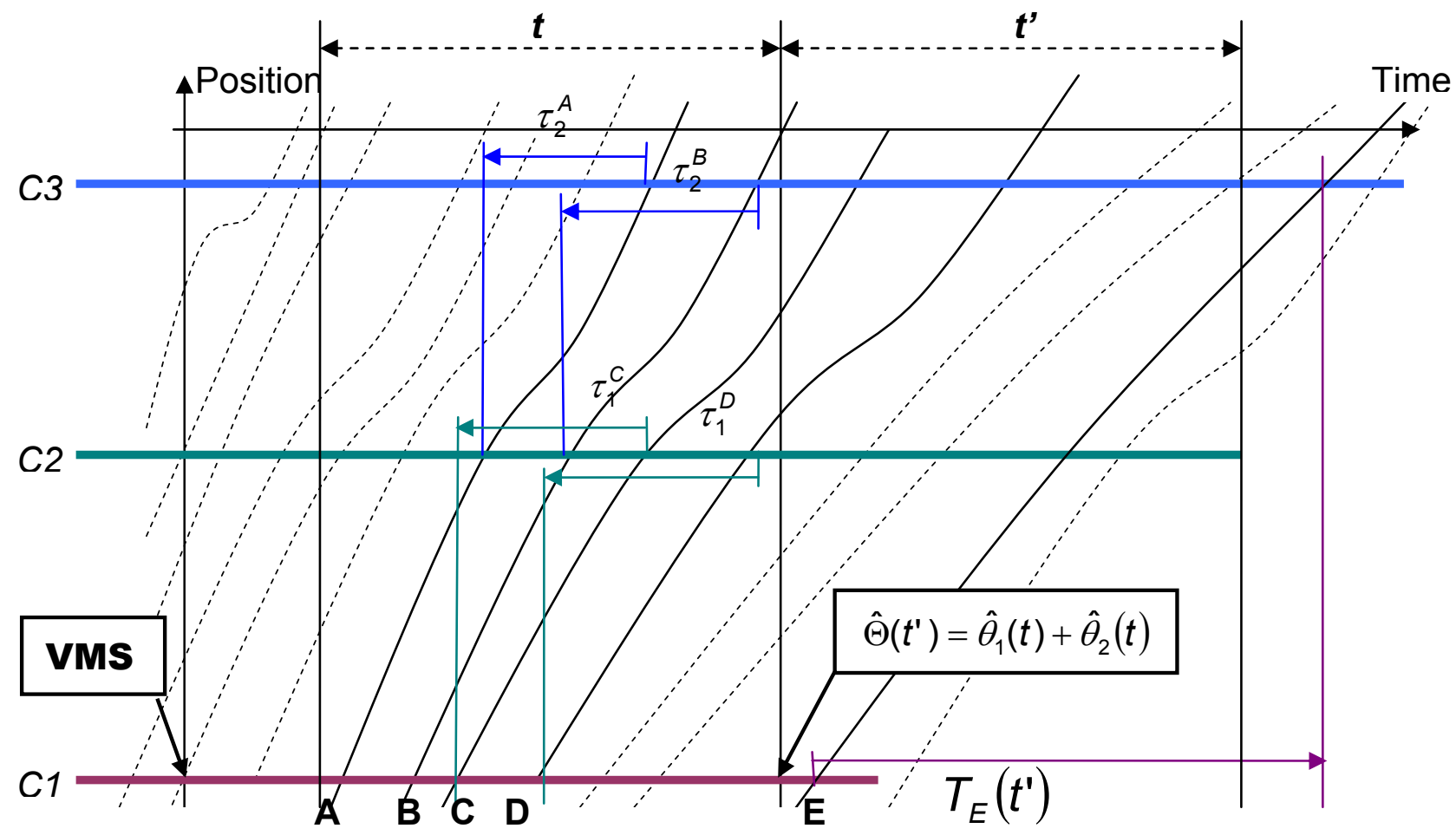

Figure 4: Representation, in the (x,t) plane, of the trajectory of some vehicles. 

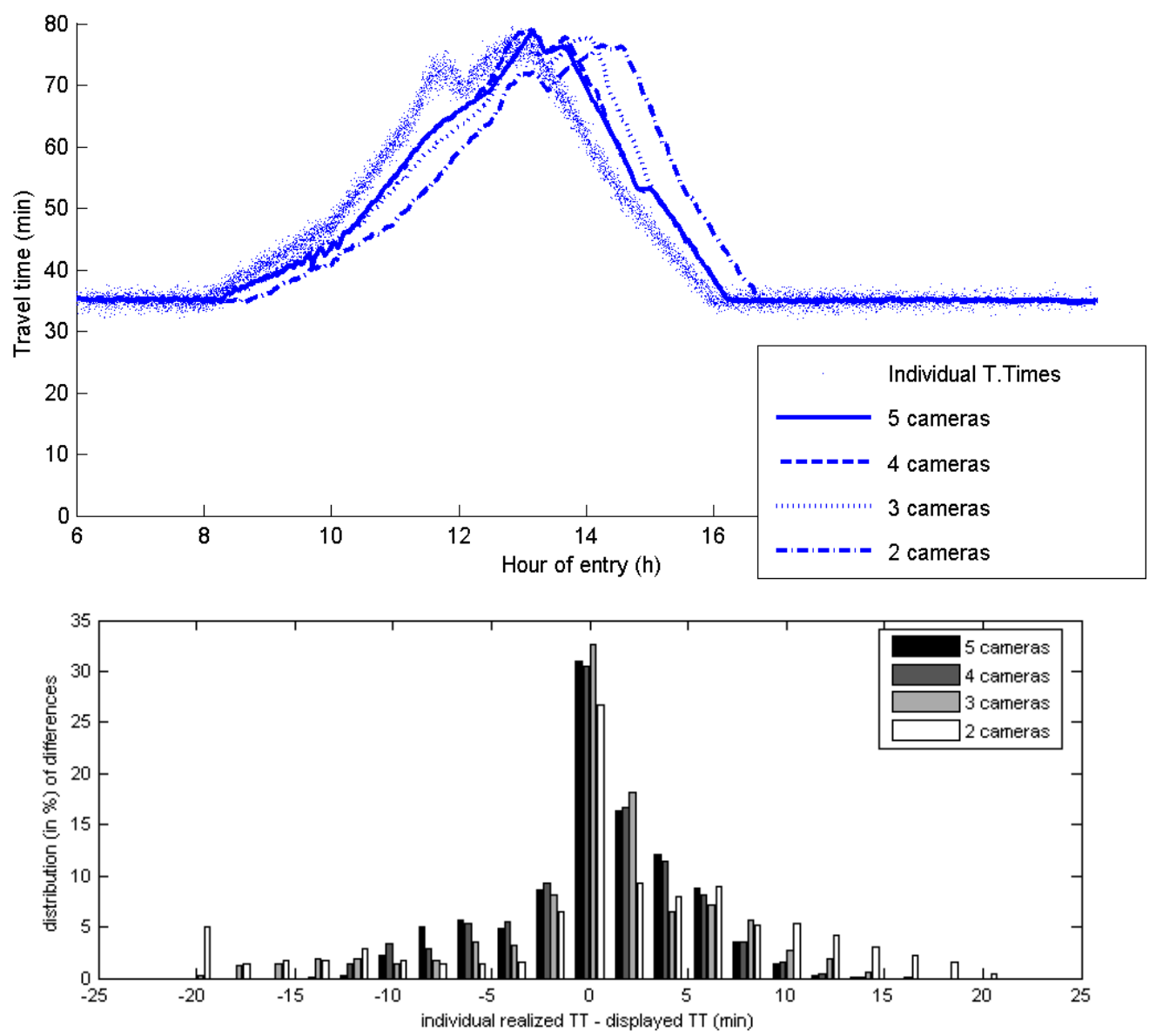

Figure 5: Results of simulation for a congested Saturday for the road travel time from Pont de Claix until Bourg d'Oisans (top); the bottom figure illustrates the distribution of discrepancies between individual realized travel times and displayed travel times for the same case. 
List of Tables:

Table 1: Results from simulation for various traffic conditions depending on the total number of cameras, for travel time information delivered at Pont de Claix until Bourg d'Oisans. 25

Table 2: Results of the model and of the in-site experiment concerning the prediction of accuracy with 2 or 3 cameras for the first subpart of the network. 


\begin{tabular}{|c|c|c|c|c|c|c|c|c|c|}
\hline \multirow{2}{*}{$\begin{array}{l}\text { Percentage of vehicles with a gap } \\
\text { between realized individual travel } \\
\text { time and travel time displayed at } \\
\text { the moment of passing bigger than }\end{array}$} & \multicolumn{3}{|c|}{ Regular Saturday } & \multicolumn{3}{|c|}{$\begin{array}{l}\text { Congested } \\
\text { Saturday }\end{array}$} & \multicolumn{3}{|c|}{$\begin{array}{c}\text { Heavily congested } \\
\text { Saturday }\end{array}$} \\
\hline & $\begin{array}{c}5 \\
\min \end{array}$ & $\begin{array}{c}10 \\
\min \end{array}$ & $\begin{array}{c}15 \\
\min \end{array}$ & $\begin{array}{c}5 \\
\min \end{array}$ & $\begin{array}{c}10 \\
\min \end{array}$ & $\begin{array}{c}15 \\
\min \end{array}$ & $\begin{array}{c}5 \\
\min \end{array}$ & $\begin{array}{c}10 \\
\min \end{array}$ & $\begin{array}{r}15 \\
\min \end{array}$ \\
\hline \multicolumn{10}{|l|}{$\begin{array}{l}\text { Depending on the total number of } \\
\text { cameras }\end{array}$} \\
\hline 5 cameras & 2.9 & 0.0 & 0.0 & 13.1 & 1.8 & 0.1 & 32.4 & 22.1 & 11.8 \\
\hline 4 cameras & 3.0 & 0.0 & 0.0 & 13.6 & 1.7 & 0.1 & 32.8 & 24.1 & 14.2 \\
\hline 3 cameras & 5.5 & 0.1 & 0.0 & 18.9 & 4.8 & 0.3 & 33.3 & 25.9 & 18.4 \\
\hline 2 cameras & 13.6 & 1.4 & 0.0 & 31.2 & 15.9 & 5.9 & 38.5 & 32.2 & 26.8 \\
\hline
\end{tabular}

Table 1: Results from simulation for various traffic conditions depending on the total number of cameras, for travel time information delivered at Pont de Claix until Bourg d'Oisans. 


\begin{tabular}{|l|c|c|}
\hline & $\begin{array}{c}2 \text { cameras } \\
\text { (Drac and } \\
\text { Cornage) }\end{array}$ & $\begin{array}{c}3 \text { cameras } \\
\text { (Drac, } \\
\text { Champagnier } \\
\text { and Cornage) }\end{array}$ \\
\hline $\begin{array}{l}\text { Predicted percentage of vehicles with more than } 10 \text { minutes of } \\
\text { error for a typical congested Saturday }\end{array}$ & $20 \%$ & $15 \%$ \\
\hline $\begin{array}{l}\text { Observed percentage of vehicles with more than } 10 \text { minutes of error } \\
\text { for the Saturday } 10^{\text {th }} \text { April } 2005 \text { (5240 vehicles studied) }\end{array}$ & $18.2 \%$ & $9.9 \%$ \\
\hline
\end{tabular}

Table 2: Results of the model and of the in-site experiment concerning the prediction of accuracy with 2 or 3 cameras for the first subpart of the network. 